\title{
Doppler assessment of pulmonary artery pressure during recovery from hyaline membrane disease
}

\author{
N J Evans, L N J Archer
}

\begin{abstract}
The changes in pulmonary artery pressure during recovery from hyaline membrane disease were studied by serial Doppler echocardiography on 37 preterm infants. Pulmonary artery pressure was assessed noninvasively by its inverse relationship with the ratio of pulmonary artery Doppler time to peak velocity and right ventricular ejection time. The pattern of rise in ratio after the acute phase of hyaline membrane disease divided into three types. In 25 infants the ratio returned to the normal range: in 12 (group 1) at the same time as the fractional inspired oxygen fell below 0.5 and in 13 (group 2) after a delay of at least 24 hours. In 12 infants (group 3) the ratio remained below the normal range during their hospital stay, suggesting pulmonary artery pressure remained high. Infants in group 3 were of significantly lower gestation and required oxygen treatment for significantly longer than infants in groups 1 and 2 . Persistently raised pulmonary pressures in group 3 may reflect lung damage either directly affecting pulmonary vasculature or exerting a secondary effect on pulmonary vascular resistance through hypoxaemia.
\end{abstract}

Pulmonary hypertension is a common and serious complication of bronchopulmonary dysplasia. ${ }^{1}$ Whether this is due to a primary effect of parenchymal lung damage sustained during mechanical ventilation or whether it is due to a secondary effect of chronic hypoxia remains unclear. High pulmonary artery pressures during the acute phase of hyaline membrane disease have been documented by both direct and indirect methods. ${ }^{2}{ }^{3}$ Whether and when pulmonary artery pressure returns to normal during the recovery period of hyaline membrane disease has not been studied.

Doppler echocardiography provides a noninvasive method to assess pulmonary artery pressure. The ratio of time to peak velocity (TPV) and right ventricular ejection time (RVET) as measured from the pulmonary artery Doppler velocity time curve has a close inverse correlation with pulmonary artery pressure. This association has been validated by comparison with direct measurements in older children and adults. ${ }^{4}$

We have previously reported the results of using these methods to study pulmonary artery pressure during the acute phase of hyaline membrane disease in a group of preterm infants. ${ }^{3}$ In this paper we describe the results of using Doppler echocardiography to assess pul- monary artery pressure in the same group of infants through the recovery period until discharge from hospital.

\section{Methods}

All infants were studied using an ATL Ultramark 4 scanner with range gated pulsed Doppler. Two dimensional imaging was done with a $7.5 \mathrm{MHz}$ transducer which incorporates a 5 $\mathrm{MHz}$ pulsed Doppler crystal.

The procedure for each study was as follows. The pulmonary artery was visualised from the parasternal long axis view. The Doppler sample volume was placed just distal to the pulmonary valve and the Doppler pattern was recorded and frozen on screen. The time intervals were measured using the incorporated Doppler measurement calipers, a print was then taken. TPV was measured as the time interval between the systolic Doppler waveform leaving the baseline and reaching its peak velocity. RVET was the time interval between the systolic waveform leaving and returning to the baseline. A mean of three systoles was taken. A second 'blind' observer repeated the measurements on 50 of the Doppler prints using a computer digitiser system. The correlation coefficient between the 'on line' and 'blind' measurements was 0.92 . The ductus arteriosus was then visualised from the high left parasternal view. The Doppler sample volume was placed in the pulmonary end of the duct and the flow pattern recorded. The duct was assessed as closed when it could not be visualised and no flow could be detected on a pulsed Doppler search of the pulmonary end of the duct.

Thirty seven preterm infants were studied. Mean gestation was 30.5 weeks (range 26-37 weeks) and mean birth weight $1570 \mathrm{~g}$ (range 720-2960 g). All infants had hyaline membrane disease, the parameters of disease severity are shown in table 1. No infant received muscle relaxants or inotropic support during the phase of the illness described in this paper. For the purposes of this study the acute phase of hyaline membrane disease was defined as the period until the fractional inspired oxygen $\left(\mathrm{FIO}_{2}\right)$ had peaked and returned to below 0.5 ; these results have been previously reported. ${ }^{3}$ The recovery phase described in this paper was the subsequent period until discharge from hospital. Infants were studied initially every 24 hours, this interval increased, as recovery continued, to approximately twice weekly. At the time of each study a record was kept of oxygen requirements, ventilator settings, and most recent blood gases. Infants were studied until either 
Table 1 Parameters of disease severity in the 37 infants

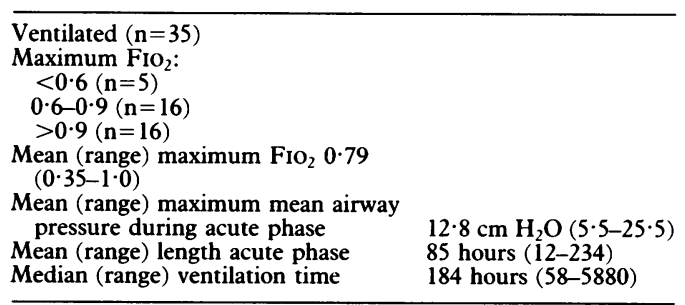

they were spontaneously breathing air and the TPV:RVET ratio was within the normal range or until they were discharged from hospital. The normal range for TPV:RVET was derived from the postnatal steady state ratio achieved in a group of 19 healthy preterm infants $(0.34$ to $0 \cdot 41)$.

Statistical analysis was by Student's $t$, MannWhitney, and Wilcoxen tests, and linear regression analysis. Significance was accepted at $p$ values of $<0.05$. The study was approved by the Central Oxford research ethics committee.

\section{Results}

In all infants TPV:RVET ratio rose in the early recovery period above the levels seen during the acute phase. The pattern of change in TPV:RVET fell into three groups. In 12 infants, group 1, the ratio rose rapidly into the normal range within 24 hours of the oxygen requirements falling (fig 1). In 13 infants, group 2 , there was a delay of more than 24 hours between the $\mathrm{FIO}_{2}$ falling to below 0.5 and the ratio rising into the normal range (fig 2 ). The

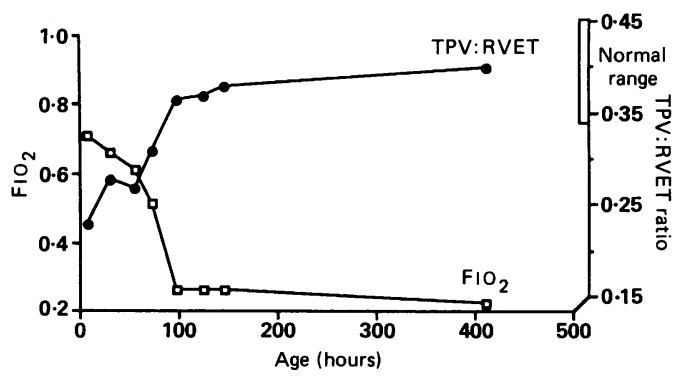

Figure 1 FIO $\mathrm{IO}_{2}$ and TPV:RVET ratio plotted against postnatal age in an infant of 27 weeks' gestation. This was typical of infants in group 1 , where the ratio rose at the same time as $\mathrm{F}_{\mathrm{IO}_{2}}$ fell.

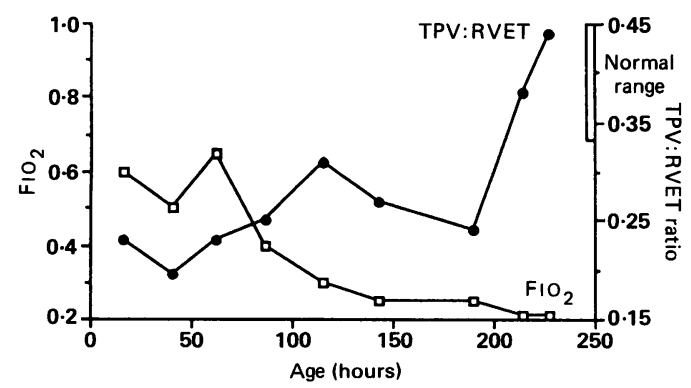

Figure $2 \mathrm{~F}_{\mathrm{IO}}$ and TPV:RVET ratio plotted against postnatal age in an infant of 30 weeks' gestation. This was typical of the infants in group 2 , where there was a delay in the rise of the ratio.

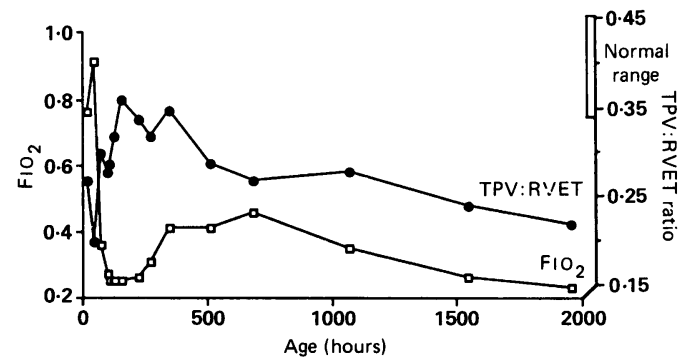

Figure $3 \mathrm{~F}_{\mathrm{IO}}$ and TPV:RVET ratio plotted against postnatat age in an infant of 28 weeks' gestation. This was typical of the infants in group 3, where the ratio did not rise into the normal range.

median delay was 103 hours with a range from 24 to 286 hours. And in 12 infants, group 3, the TPV:RVET did not rise into the normal range during the time of study (fig 3 ). The length of follow up of the infants in group 3 ranged from 336 to 5880 hours (14 to 245 days) with a median of 1380 hours (58 days). The TPV:RVET ratio taken just before discharge, or in one case death, ranged from 0.19 to 0.33 with a mean of $0 \cdot 26$. Eleven of these infants were breathing air at the time of discharge. One infant developed severe bronchopulmonary dysplasia requiring continuing ventilation in an $\mathrm{FIO}_{2}$ of 0.5 ; this infant's TPV:RVET was between 0.19 and 0.24 for most of the recovery period. He died in right heart failure at 35 weeks of age. Six of the 12 infants in this group showed a similar pattern of change in TPV: RVET as is shown in fig 3 . In the early recovery period the ratio rose towards the normal range but fell as the recovery period continued. In the other six infants, which included the infant with severe bronchopulmonary dyplasia, the ratio remained at a relatively consistent low level throughout the recovery period.

There were slight differences in the mean heart rate at the time of study. Mean (SD) heart rate for the healthy infants was 144 (15). The mean heart rates in groups 2 and 3, respectively 155 (14) and 157 (17), were higher than that in group 1 (135 (18)). Apart from between groups 2 and 3 , the differences in mean heart rate between the groups were significant, $\mathrm{p}<0.01$. There was no correlation between TPV:RVET and heart rate, either overall or within each group.

Table 2 compares variables of respiratory disease severity and maturity between the three groups. The median gestation was progressively significantly lower between the groups 1 through to 3 . Group 1 had significantly higher median birth weight than group 3. Each infant's mean acute phase ratio was significantly lower in group 2 than group 1 but was not significantly lower in group 3. The differences in time to duct closure were not significant, but groups 2 and 3 were ventilated for significantly longer than group 1. The infants in group 3 required oxygen treatment for significantly longer than groups 1 and 2 with all but two of them requiring extra inspired oxygen for more than 28 days (672 hours). 
Table 2 Maturity and clinical course in the three groups

\begin{tabular}{|c|c|c|c|c|}
\hline & $\begin{array}{l}\text { Group } 1 \\
(n=12)\end{array}$ & $\begin{array}{l}\text { Group } 2 \\
(n=13)\end{array}$ & $\begin{array}{l}\text { Group } 3 \\
(n=12)\end{array}$ & $\begin{array}{l}\text { Significance } \\
\text { (p value) }\end{array}$ \\
\hline $\begin{array}{l}\text { Median (range) gestation (weeks) } \\
\text { Median (range) birth weight (g) } \\
\text { Mean (SD) acute TPV:RVET } \\
\text { Mean (range) time duct closure noted (hours) } \\
\text { Median (range) duration ventilation (hours) } \\
\text { Median (range) time in oxygen (hours) }\end{array}$ & $\begin{array}{c}34(27-37)^{*} \\
2400(900-2960)^{*} \\
0 \cdot 27(0 \cdot 026)^{*} \\
108(24-264) \\
116(0-408)^{*} \\
189(112-408)^{*}\end{array}$ & $\begin{array}{c}30(28-34) \\
1200(900-2300) \\
0 \cdot 23(0 \cdot 032)^{*} \\
152(12-336) \\
285(74-1224)^{*} \\
170(76-1224)^{*}\end{array}$ & $\begin{array}{c}28(26-34)^{*} \\
1050(720-2350)^{*} \\
0 \cdot 25(0 \cdot 037) \\
151(13-984) \\
844(98-5880)^{*} \\
1128(260-5880)^{*}\end{array}$ & $\begin{array}{l}<0.05 \\
<0.05 \\
<0.01 \\
\text { NS } \\
<0.01 \\
<0.001\end{array}$ \\
\hline
\end{tabular}

${ }^{*}$ For each parameter there are three statistical pairings: when all are significantly different, each group has an asterisk; when two pairs are different, one pair has one and the other two asterisks.

\section{Discussion}

The two factors other than pulmonary artery pressure which will affect the TPV of pulmonary artery flow are heart rate and myocardial contractility. As the heart rate increases the TPV will be reduced. ${ }^{6}$ This effect is reduced, but not eliminated, by expressing the TPV as a ratio to RVET $^{6}$ We could find no association between heart rate and TPV:RVET within this study though there were small but significant differences between the mean heart rates of the groups of infants with hyaline membrane disease and the healthy infants. From the data of Serwer $e t a l,{ }^{6}$ we would expect the differences in mean heart rate between the groups to reflect in a change in TPV:RVET of about 0.01 . It is unlikely that this would affect the results described in this paper. Reduced myocardial contractility will prolong the TPV. Right ventricular function was not formally assessed in this study. However only the infant who died was considered to have reduced right ventricular contractility on imaging.

Eleven of the 37 infants in this study still had Doppler evidence of high pulmonary artery pressure at the time of their discharge from hospital. Despite this, at discharge these infants were breathing spontaneously in air. The prolonged need for oxygen treatment and the lower gestation of this group would suggest that these were the infants who had sustained a degree of damage to their lungs during the acute phase of their illness. Respiratory problems are common after discharge from neonatal intensive care and abnormalities of respiratory function can be detected up to 7 years of age. ${ }^{7}$ It seems likely that there is a range of damage sustained by the lungs during ventilatory support for hyaline membrane disease, with only the more severe form manifesting as a continuing recognised requirement for ventilation and oxygen treatment. Pulmonary hypertension has been well documented as a complication of these severe forms of bronchopulmonary dysplasia. ${ }^{1}$ One infant in the group that we studied developed severe bronchopulmonary dysplasia; that infant had a persistently very low TPV:RVET and died at 35 weeks of age in right ventricular failure. Our data would suggest that there is also a range of raised pulmonary vascular resistance; and that even milder degrees of lung damage, which do not require long term oxygen treatment, have continuing evidence of raised pulmonary artery pressure.

In bronchopulmonary dysplasia some of the raised pulmonary vascular resistance probably results directly from parenchymal lung damage but there is evidence that it may in part be a secondary effect of hypoxia. ${ }^{1}$ Hypoxia is a potent pulmonary vasoconstrictor and, if chronic, may lead to alteration in the structure of the pulmonary vascular bed. Such changes have been seen at necropsy in cases of bronchopulmonary dysplasia. ${ }^{8}$ In addition, catheter studies in infants with severe bronchopulmonary dysplasia have shown that in many cases pulmonary artery pressure will fall if $100 \%$ oxygen is inspired.' In half the infants in group 3 of our study, the TPV:RVET fell as the recovery period continued. It seems possible that borderline low levels of oxygen or unrecognised hypoxaemic periods may be sustaining high pulmonary artery pressure in those infants. Pulse oximetry provides a good non-invasive means to monitor oxygenation in those infants. ${ }^{9}$ It may be that continuous monitoring of infants who have had a prolonged need for oxygen treatment should become routine, even after transfer into air. The presence of a low TPV:RVET ratio may provide a further pointer to those infants in whom particularly close attention to oxygenation is needed. Further study is required to clarify those points.

Further study is also needed to follow up a group of these infants beyond their discharge from hospital. Firstly, to establish whether and when the ratio returns to the normal range and secondly, to see if a low ratio is associated with a higher risk of postneonatal respiratory problems.

We thank Dr Andrew Wilkinson and Dr Peter Hope for their help and encouragement and for allowing us to study infants under their care. We also thank Sheila Hayes for typing the manuscript.

1 Goodman G, Perkins RM, Anas NG, et al. Pulmonary hypertension in infants with bronchopulmonary dysplasia. tension in infants with
$\mathcal{f}$ Pediatr 1988;112:67-72.

2 Moss AJ, Emmanouilides GC, Petteri O, Higashino SM, Adams FH. Postnatal circulatory and metabolic adjustments in normal and distressed premature infants. Bio Neonate 1965;8:177-97.

3 Evans NJ, Archer LNJ. Doppler assessment of pulmonary artery pressure and extra-pulmonary shunting in the acute phase of hyaline membrane disease. Arch Dis Child 1991; 66:6-11.

4 Kitabatake A, Inoue $M$, Asao $M$, et al. Non-invasive evaluation of pulmonary hypertension by a pulsed Doppler technique. Circulation 1983;68:302-9.

5 Evans NJ, Archer LNJ. Postnatal circulatory adaptation in healthy term and preterm newborns. Arch Dis Child 1990; 65:24-6.

6 Serwer GA, Cougle AG, Eckerd JM, Armstrong BE. Factors affecting use of the Doppler-determined time from flow onset to maximal pulmonary artery velocity for measurement of pulmonary artery pressure in children. Am ment of pulmonary ar $1986 ; 58: 352-6$

7 Chan KN, Noble-Jamieson CM, Elliman A, Bryan EM, Silverman $M$. Lung function in children of low birthweight. Arch Dis Child 1989;64:1284-93.

8 Tomashefski JF, Oppermann HC, Vawter GF, Reid LM. Bronchopulmonary dysplasia: a morphometric study with Bronchopulmonary dysplasia: a morphometric study with
emphasis on the pulmonary vasculature. Pediatr Pathol 1984;2:469-87.

9 Southall DP, Bignall S, Stebbens VA, et al. Pulse oximeter and transcutaneous arterial oxygen measurements in neonatal and paediatric intensive care. Arch Dis Child 1987;62 882-8. 